Article

\title{
Data Acquisition Methodologies Utilizing Ground Penetrating Radar for Cassava (Manihot esculenta Crantz) Root Architecture
}

\author{
Alfredo Delgado ${ }^{1, *}$, Alexandre Novo ${ }^{1}$ and Dirk B. Hays ${ }^{2}$ \\ 1 IDS GeoRadar North America, Golden, CO 80401, USA; alexandre.novo@idsgeoradar.com \\ 2 Soil and Crop Sciences, Texas A\&M University, College Station, TX 77843-2474, USA; dbhays@tamu.edu \\ * Correspondence: alfredo.delgado@idsgeoradar.com; Tel.: +1-303-232-3047
}

Received: 5 March 2019; Accepted: 10 April 2019; Published: 15 April 2019

\begin{abstract}
Cassava (Manihot esculenta Crantz), a root crop utilized as food and industrial starch product, develops and maintains its marketable product sub-surface. Often, however, it is difficult to determine the potentially marketable goods available at any given time due to the sub-surface nature of the product and the inability to non-destructively sample. This dilemma has provided an avenue for application of ground penetrating radar. Relatively available designs of this technology, however, are cumbersome and do not provide the efficiencies for field applications. The objective of this research was to determine the functionality of a two Gigahertz frequency IDS GeoRadar C-Thrue antenna for the detection and parameterization of root architecture to be utilized for estimating marketable product. Cassava roots were buried across three horizontal and two vertical orientations to simulate the multi-directional nature of cassava roots. The antenna has dual polarization which also allowed to testing efficacy of polarization for detecting the varying root orientations. This study found that the C-Thrue system, more specifically, the Vertical transmit and Vertical receive polarization, was the most effective at accurately estimating cassava root length and widths at varying angles that simulate root development in true fields.
\end{abstract}

Keywords: Ground Penetrating Radar; Root Architecture; Cassava

\section{Introduction}

Cassava (Manihot esculenta Crantz) is a tropical root crop originally from South America and serves as a staple food source for an estimated 800 million people [1]. More than a tenth of the world's population relies on this food source, and in tropical countries, it follows only maize and rice in caloric intake [2]. Worldwide, cassava is the second most important source of starch after maize [3]. Between 1991-1993 and 2011-2013, the global harvested area of cassava expanded by 25\%, from 16.5 million to 20.7 million hectares, which was the biggest percentage increase among the world's five major food crops. Most of this cultivated increase occurred in Africa (with an increase of 39.2\%), which alone produces nearly 145 million metric tons of cassava per year while South East (SE) Asia's (particularly, Thailand, Cambodia, and Vietnam) average fresh root yields have almost doubled in the last 20 years [4]. In SE Asia, cassava is mostly an industrial crop used for the production of starch and dried chips which allow the breeders to concentrate basically on high fresh root yield, high dry matter content, and adequate plant architecture [3,5]. Strong markets in SE Asia encourage the adoption of new technologies (e.g., highly productive varieties and appropriate cultivation practices).

This improvement would be expected to occur if new traits for root architecture can be incorporated into breeding programs and tools for early selection of marketable material are available. However, limited information on growth patterns in cassava roots as compared to aboveground biomass exists [6]. 
This is a serious constraint considering that root is the main commercial product. This limitation and the need to perform destructive samplings restrict the farmers' ability to screen root development through the growing season. As such, cassava researchers and farmers need new rapid, non-destructive procedures to capture root traits [7].

Many technologies currently exist and are utilized for non-destructive root characterization. Some can include minirhizotrons, computed tomography, and magnetic resonance imaging [8-10]. However, these technologies are currently limited by time requirements and costs for implementing these tools. Ground penetrating radar (GPR) is an existing, affordable, and rapidly evolving technology. GPR has often been utilized as a small cross-section near-surface object detection tool [11-14]. GPR has been utilized to non-destructively image coarse root biomass and architecture in various tree and shrub species [15-28]. More recently, GPR has demonstrated that it can be used as a high throughput (HT), non-destructive, three-dimensional imaging method for quantifying cassava root mass [29]. Most GPR systems work in a time domain function by emitting electromagnetic pulses into the ground in which part of the energy is reflected, transmitted, or scattered at boundaries of contrasting materials [30,31]. The reflected strength of the return is recorded as a function of two-way travel time [31]. Many thousands of measurements are acquired across a planned grid network by moving the antenna along a ground transect at fixed intervals. These returns can be quantified and rendered into a 3D field allowing for visualization and mapping of near-surface root biomass.

We hypothesize that current technology can detect and measure root features and root orientation parameters. By utilizing a controlled in-vitro environment, we can reduce the unexplained variance in data capture and enhance the sampling protocol to more accurately detect rooting parameters that are necessary for estimating cassava size and shape necessary for decision support in breeding programs and to identify marketable materials near real-time.

\section{Materials and Methods}

The unit utilized for this study was the IDS GeoRadar C-Thrue system [32]. This unit is of compact design that is fully integrated into one handheld unit (antenna, computer, control unit, and battery). This system design is considered optimal for maneuverability and access to closed canopy rows often found in cassava fields. The C-Thrue design was originally developed for concrete scanning in civil applications. The system utilizes a dual polarization for multi-level detection, which is optimal for root architecture in that some roots may overlap and root orientations can vary. The specific polarizations are horizontal transmit and horizontal receive $(\mathrm{HH})$ and vertical transmit and vertical receive (VV). The system is ground coupled and allows for minimized signal dispersion at the surface. Acquisition parameters for the sensor were set at $1 \mathrm{scan}$ per $0.2 \mathrm{~cm}$ and a time window of $12 \mathrm{~ns}$ and 512 samples per scan.

To reduce the error incurred in gridded data acquisition by human interaction, a computer numerical control (CNC) machine was utilized. This would allow for precision in instrument alignment and movement when defining sampling protocols. The CNC machine utilized is accurate to within $0.01 \mathrm{~cm}$ over this area and moves at speed ranging from $0.1 \mathrm{~cm} / \mathrm{s}$ to $10 \mathrm{~cm} / \mathrm{s}$ (Figure 1). A controlled test site was utilized for this experiment to maintain a high level of uniformity in data acquisition. For this study, it was necessary to control environmental variables, such as soil variability and soil moisture, so that optimization of system sampling protocol can occur for only root variability. The test site was in the form of a rectangular sandbox that measures $400 \mathrm{~cm}$ long by $300 \mathrm{~cm}$ wide by $110 \mathrm{~cm}$ deep. Base and walls are constructed of untreated plywood and assembled together with stainless steel screws and high weight capacity straps. To secure high fidelity of repeated trials for replication, the test area was covered in a carbon-based microwave absorber (PPG Aerospace-Cuming Microwave). This material reduces electromagnetic interference and repetitive backscatter from sandbox bounds. This component is a flexible carbon loaded microwave and radar absorbing material specifically designed to better absorb the range of frequencies that were utilized in this study. 


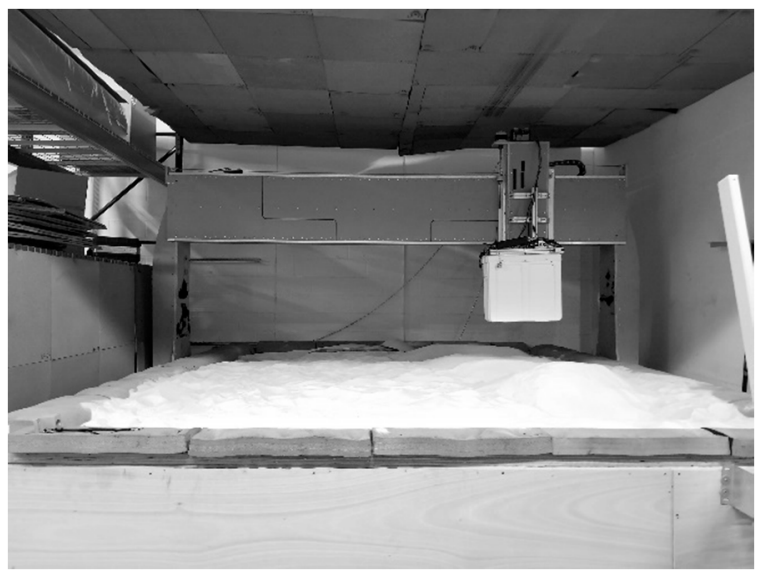

Figure 1. Image of Computer Numerical Control unit over test sandbox.

Full-resolution GPR data acquisition has demonstrated a high level of near-surface object visualization $[33,34]$. For this reason, the minimum allowable cross-line spacing was utilized. This cross-line spacing was set to $2.5 \mathrm{~cm}$. Full-resolution for this system as determined utilizing the Nyquist Theorem exceeds this value. The theoretical full-resolution was derived by estimating wavelength $(\lambda)$ which is defined by Equation (1):

$$
\lambda=\left(\frac{c}{\sqrt{\varepsilon_{r}}}\right) / f
$$

where the media utilized had a relative dielectric permittivity $\left(\varepsilon_{r}\right)$ of 4.5 , frequency $(f)$ of our system was $2 \mathrm{GHz}$, and $c$ is the speed of light $(30 \mathrm{~cm} / \mathrm{ns})$. Therefore, a quarter of the wavelength for this host material was $1.8 \mathrm{~cm}$ when velocity is calculated at $14 \mathrm{~cm} / \mathrm{ns}$. However, the cross-line spacing that was set at $2.5 \mathrm{~cm}$ was due to a structural limitation in the system in which the wheel spacing of the unit is $18 \mathrm{~cm}$. Since the wheels require enough contact for encoder functionality, a gridded platform was constructed with notches (Figure 2) to minimize encoder slippage that would occur by solely having contact with the sand. This adjustment, therefore, limited the minimum spacing between scans to $2.5 \mathrm{~cm}$ to allow for equal interval passes without encoder slippage.

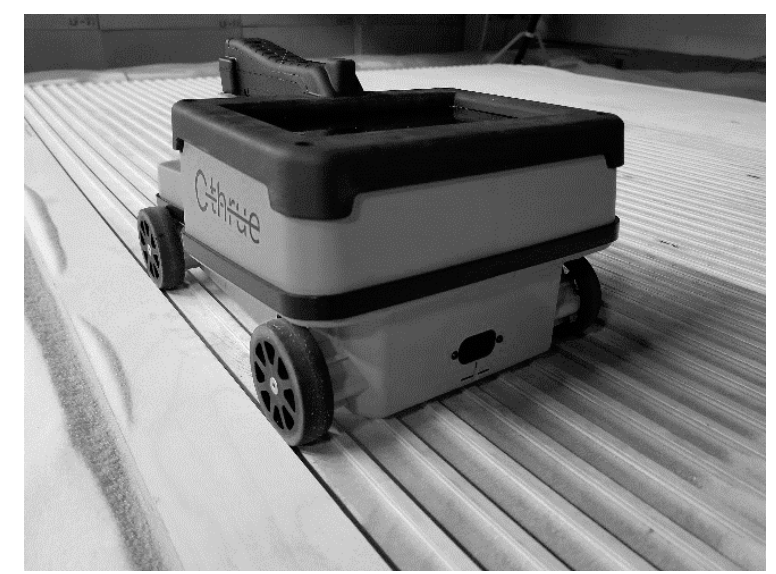

Figure 2. Illustration of $\mathrm{C}$-Thrue sensor on the gridded platform to limit encoder slippage. The distance between the center of each wheel track is $2.5 \mathrm{~cm}$. The sensor dimensions are $20 \mathrm{~cm}$ wide by $30 \mathrm{~cm}$ long.

Roots were chosen based on three size classes (Small, Medium, Large) with three roots per class. This would allow for estimating accuracy at each class and sensitivity to size characteristics. These characteristics can be found in Table 1. Measurements were taken for total root length, and three 
root diameters were taken at equal intervals across the root to account for root tapering. Figure 3 provides an illustration of the measurement procedure.

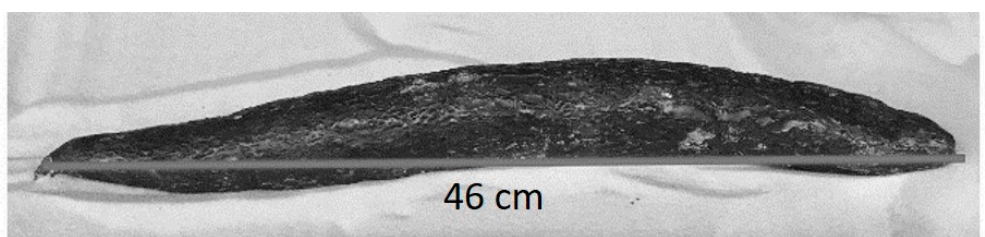

(a)

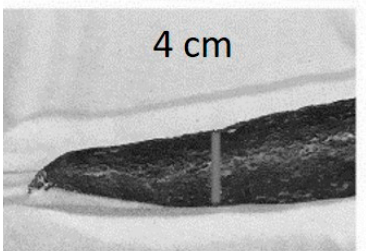

(b)

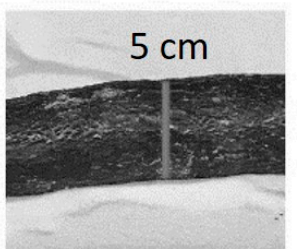

(c)

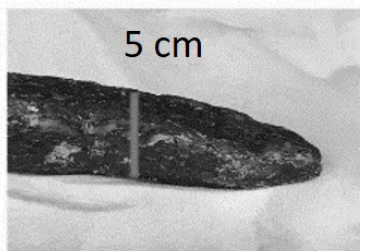

(d)

Figure 3. Illustration of root measurement procedure: (a) Root Length; (b) Root Width 1 ; (c) Root Width 2; (d) Root Width 3.

Table 1. Dimensions of all roots utilized in the study. Measurements were derived by taking the full root length and segmenting into three sections; each section diameter was then measured to obtain width value.

\begin{tabular}{ccccc}
\hline Root Number & Length & Width 1 & Width 2 & Width 3 \\
\hline \multicolumn{5}{c}{ Large Root Classification } \\
\hline Root 1 & $38 \mathrm{~cm}$ & $6 \mathrm{~cm}$ & $9 \mathrm{~cm}$ & $8 \mathrm{~cm}$ \\
Root 2 & $36 \mathrm{~cm}$ & $5 \mathrm{~cm}$ & $8 \mathrm{~cm}$ & $9 \mathrm{~cm}$ \\
Root 3 & $46 \mathrm{~cm}$ & $4 \mathrm{~cm}$ & $5 \mathrm{~cm}$ & $5 \mathrm{~cm}$ \\
\hline \multicolumn{5}{c}{ Medium Root Classification } \\
\hline Root 4 & $37 \mathrm{~m}$ & $4 \mathrm{~cm}$ & $5 \mathrm{~cm}$ & $5 \mathrm{~cm}$ \\
Root 5 & $35 \mathrm{~m}$ & $4 \mathrm{~cm}$ & $5 \mathrm{~cm}$ & $5 \mathrm{~cm}$ \\
Root 6 & $30 \mathrm{~m}$ & $4 \mathrm{~cm}$ & $6 \mathrm{~cm}$ & $5 \mathrm{~cm}$ \\
\hline \multicolumn{5}{c}{ Small Root Classification } \\
\hline Root 7 & $25 \mathrm{~m}$ & $4 \mathrm{~cm}$ & $4 \mathrm{~cm}$ \\
Root 8 & $20 \mathrm{~m}$ & $4 \mathrm{~cm}$ & $5 \mathrm{~cm}$ & $4 \mathrm{~cm}$ \\
Root 9 & $20 \mathrm{~m}$ & $3 \mathrm{~cm}$ & $6 \mathrm{~cm}$ \\
\hline
\end{tabular}

Root orientation in true fields is omnidirectional and, for this reason, efforts were taken to determine the sensor efficacy with multiple root orientations. Each of the nine roots was buried in two vertical positions relative to the surface (0-degrees \& 45-degrees) as well as three horizontal directions relative to the scan direction (parallel to scan, perpendicular to scan, and a 45-degree angle to scan). Roots were buried in the sandbox at a depth similar to that of an actively growing root in the field. More specifically, if the root was buried parallel to the surface, it was buried at $15 \mathrm{~cm}$ from the surface, and when roots were at a 45-degree angle to the surface, they were buried at $0 \mathrm{~cm}$ from the surface and continued downwards to a maximum depth relative to the root length. Visual representation of the root orientation parameters and burying conditions can be found in Figure 4. 
Vertical Positions

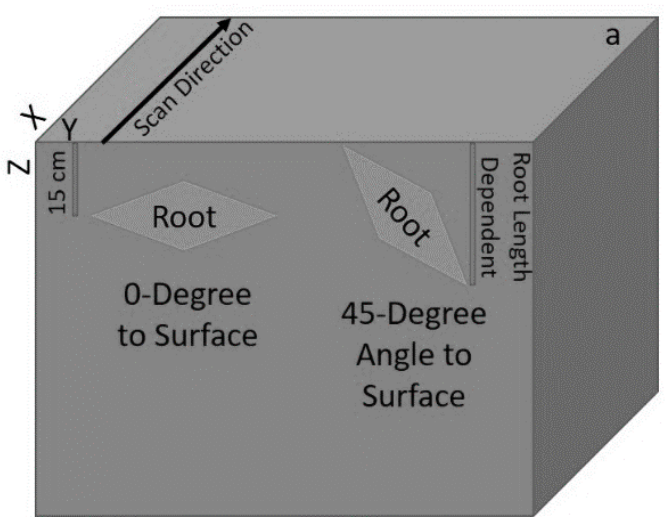

Horizontal Positions

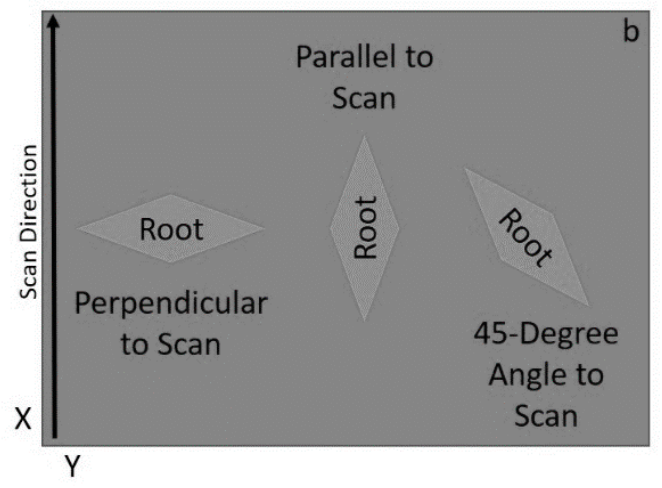

Figure 4. Illustration of root planting parameters: (a) 3D representation of the sandbox: Vertical position in relation to the surface (0-degree to surface and 45-degrees to surface). Roots at 0-degree to the surface were buried at $15 \mathrm{~cm}$ depth, while roots at 45 -degrees to surface were buried starting at surface and continuing downward to the max depth dependent on root length. (b) Plan (Top) view of the sandbox: Horizontal position in relation to the scan direction. There were three root positions being perpendicular to the scan direction, parallel to the scan direction, and a 45-degree angle to the scan direction.

The data pre-processing procedure was performed using GPR-Slice software [35]. The data was processed in GPR-Slice, which allows for three-dimensional rendering and volume extraction utilizing an OpenGL interface. Before the export of root volumes, a pre-processing procedure was performed to create the isosurfaces. This process required a signal gain correction, which was followed by filtering of the raw radargrams for noise removal using a bandpass filter (Low: $500 \mathrm{MHz} / \mathrm{High}$ : $5000 \mathrm{MHz}$ ) followed by a median background filter. Median background filters provide better filtering than standard average scan-background filtering-as the peak responses from cassava reflections would not overweight a median scan used in this subtraction filter. Kirchhoff migrations were then performed on the background filtered data to migrate hyperbolic responses and to collapse diffractions. The previously calculated $14 \mathrm{~cm} / \mathrm{ns}$ propagation velocity generated satisfactory results and was also used for time-to-depth conversion of the data. The migrated image was then converted using a Hilbert transform to rectify the pulse data into the pulse envelope. The sinusoidal pulses were converted to positive domain envelopes. This was accomplished by conducting a Fourier Transform to convert the radargrams to the frequency domain and shifting the imaginary frequencies by 90 -degrees followed by an inverse Fourier Transform. This is useful to define regions of just strong or weak reflections as the signal is completely rectified in the positive domain. We then began a depth- slice process utilizing the Slice XYZ function in GPR-Slice. Data were sampled at 12 ns per scan; however, relative data to the root zone was only found between $1.07 \mathrm{~ns}$ and $6.07 \mathrm{~ns}$. Therefore, $5 \mathrm{~ns}$ was sliced into 15 -time slices at $25 \%$ overlap between slice for an estimate time slice depth of $0.54 \mathrm{~ns}$. The data then underwent a gridding process to create 2D horizontal images of the slices at different depths in which data are interpolated to fill gaps between radargrams utilizing an inverse distance weighting function. Volume renderings were developed from the $2 \mathrm{D}$ depth-slices which were interpolated vertically to enhance $3 \mathrm{D}$ visualization creating more images between depth levels (Figure 5a). 


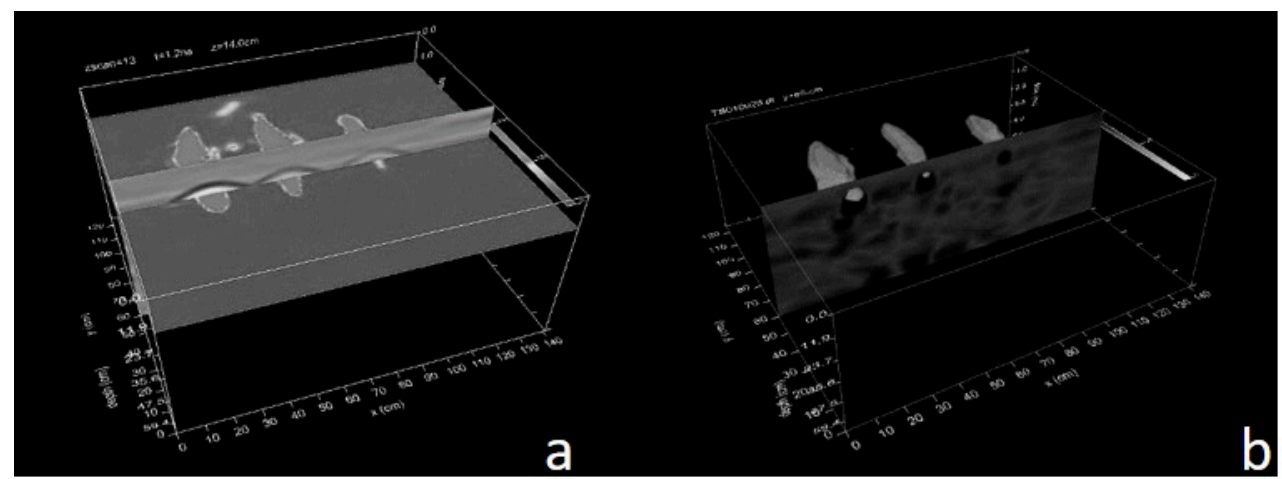

Figure 5. Illustration of: (a) time slice and associated filtered radargram; (b) isosurfaces derived utilizing Hilbert processed radargram data.

These interpolation methods may have some effect on the $\mathrm{X}, \mathrm{Y}$ dimension of the root feature. Finally, the isosurface renderings were generated to extract a volumetric body, which represents the shapes and dimensions of the targets. An amplitude threshold value defines a maximum number of possible surfaces within the three-dimensional volume, which are automatically calculated. Then, any surface with a value equal to or greater than $70 \%$ of the maximum amplitude value (threshold) was displayed. Therefore, isosurface functions may affect the dimensions of the root feature (Figure $5 b$ ). These isosurface volumes were measured for accuracy assessment relative to true root dimensions. This procedure was done by exporting the isosurfaces as graphical tins that could be rendered and characterized in AutoCAD 2012 [36]. The logic block diagram in Figure 6 provides context on the processing flow discussed here.

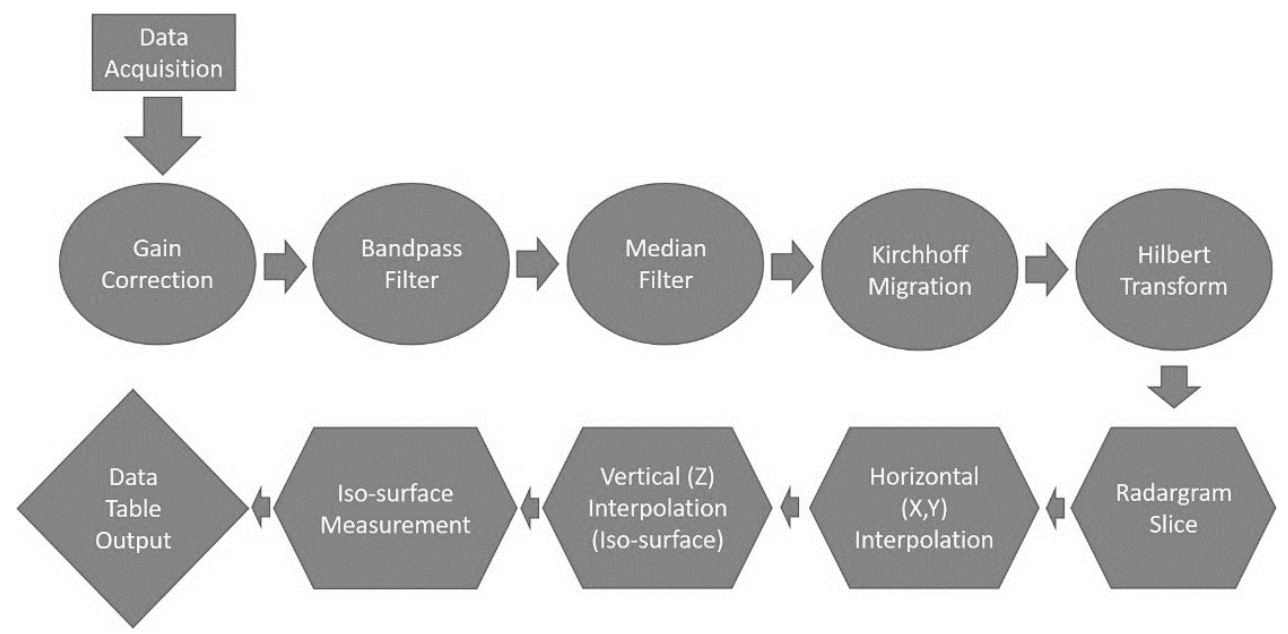

Figure 6. Flow diagram of pre-processing and processing steps for data extraction.

\section{Results}

The following results are presented as an analysis of accuracy by size class as established in the methods. This was done since the objective of the article was to establish what are the capabilities of a current sensor for detecting root size and orientation. Size class large references data were obtained from roots 1,2, and 3. Size class medium reference data were obtained from roots 4, 5, and 6 . Size class small references data were obtained from roots 7,8 , and 9 . The accuracy analysis was conducted utilizing standard root mean squared error (Equation (2)). Where $P i$ is the predicted value, $O i$ is the observed value, and $n$ is the number of samples.

$$
R M S E=\sqrt{\sum \frac{(P i-O i)^{2}}{n}}
$$


Also utilized was a standard deviation value to understand the precision of the measurement. This would allow for greater confidence in defining parameters. The standard deviation was calculated using Equation (3), where $P i$ is the predicted sample value, $\overline{P i}$ is the mean predicted sample value, and $n$ is the number of samples.

$$
S D=\sqrt{\sum \frac{(P i-\overline{P i})^{2}}{n}}
$$

The results are broken down into three categories. These were Cross-line spacing, Polarization, and Depth. Depth analysis was accomplished by analyzing the differences between Width 1 , Width 2, and Width 3 as each measurement would be located at different depths since the root was buried at a 45-degree angle to surface.

\subsection{Cross-Line Spacing}

It is defined as the spatial distance between two radargrams. From the original dense $(2.5 \mathrm{~cm}$ cross-line spacing) data set, a decimation study was conducted. The purpose of this study was to identify the maximum spacing allowable for accurate analysis. This information could be utilized to modify future antenna array designs to minimize the expense and size of future units. Three data sets with three different cross-line spacing values were compared: $2.5 \mathrm{~cm}, 5 \mathrm{~cm}$, and $10 \mathrm{~cm}$. As $2 \mathrm{GHz}$ dual polarization antenna arrays with a $10 \mathrm{~cm}$ cross-line spacing are already commercially available [37], we did not continue decimating further than that. In-line spacing was kept constant at $0.2 \mathrm{~cm}$ as set by the antenna acquisition software configuration.

When analyzing this data across all variables, it was found that the cross-line spacing decimation until $10 \mathrm{~cm}$ did not create a significant difference in accuracy for both root length and root width for small, medium, or large root classes. However, in most instances, the standard deviation increased with cross-line spacing. These results can be found in Table 2.

Table 2. Cross-line spacing for root length measures. The root mean squared error (RMSE) and standard deviation (SD) for each root category are provided.

\begin{tabular}{|c|c|c|c|}
\hline \multicolumn{4}{|c|}{ Results of Cross-Line Spacing Decimation for three Root Size Classes } \\
\hline \multicolumn{4}{|c|}{ Root Length } \\
\hline & Small & Medium & Large \\
\hline Cross-line Spacing & RMSE SD & RMSE SD & RMSE SD \\
\hline $2.5 \mathrm{~cm}$ & $9.0 \mathrm{~cm} \pm 2.4 \mathrm{~cm}$ & $8.4 \mathrm{~cm} \pm 4.8 \mathrm{~cm}$ & $6.3 \mathrm{~cm} \pm 2.4 \mathrm{~cm}$ \\
\hline $5 \mathrm{~cm}$ & $7.1 \mathrm{~cm} \pm 1.9 \mathrm{~cm}$ & $9.7 \mathrm{~cm} \pm 6.5 \mathrm{~cm}$ & $6.4 \mathrm{~cm} \pm 3.4 \mathrm{~cm}$ \\
\hline $10 \mathrm{~cm}$ & $7.5 \mathrm{~cm} \pm 1.4 \mathrm{~cm}$ & $10.9 \mathrm{~cm} \pm 7.9 \mathrm{~cm}$ & $4.8 \mathrm{~cm} \pm 5.2 \mathrm{~cm}$ \\
\hline \multicolumn{4}{|c|}{ Root Width } \\
\hline & Small & Medium & Large \\
\hline Cross-line Spacing & RMSE SD & RMSE SD & RMSE SD \\
\hline $2.5 \mathrm{~cm}$ & $7.9 \mathrm{~cm} \pm 2.3 \mathrm{~cm}$ & $5.6 \mathrm{~cm} \pm 2.3 \mathrm{~cm}$ & $9.6 \mathrm{~cm} \pm 2.5 \mathrm{~cm}$ \\
\hline $5 \mathrm{~cm}$ & $6.9 \mathrm{~cm} \pm 2.7 \mathrm{~cm}$ & $6.5 \mathrm{~cm} \pm 2.3 \mathrm{~cm}$ & $9.9 \mathrm{~cm} \pm 2.2 \mathrm{~cm}$ \\
\hline $10 \mathrm{~cm}$ & $7.7 \mathrm{~cm} \pm 3.3 \mathrm{~cm}$ & $6.9 \mathrm{~cm} \pm 3.0 \mathrm{~cm}$ & $11.9 \mathrm{~cm} \pm 3.2 \mathrm{~cm}$ \\
\hline
\end{tabular}

\subsection{Polarization}

Since cross-line spacing decimation up to $10 \mathrm{~cm}$ was not found to create significant differences in accuracy, the analysis for Polarization was performed utilizing solely the $2.5 \mathrm{~cm}$ cross-line spacing. For this section, the results are constructed based on the fact that polarization, in theory, would be affected by root orientation. Each root across the three size classes was buried in all orientations to include vertical and horizontal to surface and parallel, perpendicular, and angled to scan direction. This would allow for sampling like that of real field root orientation. The results of the analysis can be found in Table 3. 
Table 3. Results of accuracy analysis utilizing root mean squared error (RMSE) and standard deviation (SD) for identifying Polarization response to orientation across three root size classes. Asterisks signify no data available for that parameter due to the inability to segment root features from isosurfaces.

\begin{tabular}{|c|c|c|c|c|c|c|}
\hline \multicolumn{7}{|c|}{ Polarization Response to Orientation } \\
\hline \multicolumn{7}{|c|}{ VV Polarization } \\
\hline \multicolumn{7}{|c|}{ Root Length } \\
\hline & \multicolumn{3}{|c|}{ Horizontal to Surface } & \multicolumn{3}{|c|}{ Vertical to Surface } \\
\hline & Small & Medium & Large & Small & Medium & Large \\
\hline & RMSE SD & RMSE SD & RMSE SD & RMSE SD & RMSE SD & RMSE SD \\
\hline Angle & $4.7 \mathrm{~cm} \pm 2.1 \mathrm{~cm}$ & $3.3 \mathrm{~cm} \pm 2.7 \mathrm{~cm}$ & $2.3 \mathrm{~cm} \pm 1.4 \mathrm{~cm}$ & $11.1 \mathrm{~cm} \pm 3.8 \mathrm{~cm}$ & $8.4 \mathrm{~cm} \pm 6.2 \mathrm{~cm}$ & $6.8 \mathrm{~cm} \pm 4.7 \mathrm{~cm}$ \\
\hline Parallel & $3.1 \mathrm{~cm} \pm 2.5 \mathrm{~cm}$ & $4.6 \mathrm{~cm} \pm 3.4 \mathrm{~cm}$ & $3.1 \mathrm{~cm} \pm 2.8 \mathrm{~cm}$ & * & * & * \\
\hline Perpendicular & $5.8 \mathrm{~cm} \pm 5.2 \mathrm{~cm}$ & $2.1 \mathrm{~cm} \pm 2.6 \mathrm{~cm}$ & $4.2 \mathrm{~cm} \pm 3.1 \mathrm{~cm}$ & $8.8 \mathrm{~cm} \pm 1.5 \mathrm{~cm}$ & $9.1 \mathrm{~cm} \pm 2.5 \mathrm{~cm}$ & $7.9 \mathrm{~cm} \pm 2.5 \mathrm{~cm}$ \\
\hline \multicolumn{7}{|c|}{ Root Width } \\
\hline & \multicolumn{3}{|c|}{ Horizontal to Surface } & \multicolumn{3}{|c|}{ Vertical to Surface } \\
\hline & Small & Medium & Large & Small & Medium & Large \\
\hline & RMSE SD & RMSE SD & RMSE SD & RMSE SD & RMSE SD & RMSE SD \\
\hline Angle & $1.2 \mathrm{~cm} \pm 1.2 \mathrm{~cm}$ & $2.6 \mathrm{~cm} \pm 1.5 \mathrm{~cm}$ & $1.8 \mathrm{~cm} \pm 1.5 \mathrm{~cm}$ & $5.8 \mathrm{~cm} \pm 1.5 \mathrm{~cm}$ & $9.7 \mathrm{~cm} \pm 3.7 \mathrm{~cm}$ & $5.3 \mathrm{~cm} \pm 2.1 \mathrm{~cm}$ \\
\hline Parallel & $3.1 \mathrm{~cm} \pm 2.5 \mathrm{~cm}$ & $6.0 \mathrm{~cm} \pm 2.3 \mathrm{~cm}$ & $19.1 \mathrm{~cm} \pm 2.6 \mathrm{~cm}$ & $*$ & $*$ & $*$ \\
\hline Perpendicular & $1.4 \mathrm{~cm} \pm 0.6 \mathrm{~cm}$ & $4.2 \mathrm{~cm} \pm 3.2 \mathrm{~cm}$ & $3.9 \mathrm{~cm} \pm 1.5 \mathrm{~cm}$ & $3.1 \mathrm{~cm} \pm 1.0 \mathrm{~cm}$ & $4.8 \mathrm{~cm} \pm 1.5 \mathrm{~cm}$ & $3.7 \mathrm{~cm} \pm 2.6 \mathrm{~cm}$ \\
\hline \multicolumn{7}{|c|}{ HH Polarization } \\
\hline \multicolumn{7}{|c|}{ Root Length } \\
\hline & \multicolumn{3}{|c|}{ Horizontal to Surface } & \multicolumn{3}{|c|}{ Vertical to Surface } \\
\hline & Small & Medium & Large & Small & Medium & Large \\
\hline & RMSE SD & RMSE SD & RMSE SD & RMSE SD & RMSE SD & RMSE SD \\
\hline Angle & $6.7 \mathrm{~cm} \pm 1.2 \mathrm{~cm}$ & $5.2 \mathrm{~cm} \pm 2.1 \mathrm{~cm}$ & $3.5 \mathrm{~cm} \pm 2.0 \mathrm{~cm}$ & $7.4 \mathrm{~cm} \pm 4.0 \mathrm{~cm}$ & $9.3 \mathrm{~cm} \pm 3.8 \mathrm{~cm}$ & $11.5 \mathrm{~cm} \pm 7.6 \mathrm{~cm}$ \\
\hline Parallel & $10.9 \mathrm{~cm} \pm 8.1 \mathrm{~cm}$ & $8.7 \mathrm{~cm} \pm 7.3 \mathrm{~cm}$ & $16.5 \mathrm{~cm} \pm 9.6 \mathrm{~cm}$ & $*$ & $*$ & $*$ \\
\hline Perpendicular & $12.3 \mathrm{~cm} \pm 0.6 \mathrm{~cm}$ & $10.0 \mathrm{~cm} \pm 1.9 \mathrm{~cm}$ & $9.2 \mathrm{~cm} \pm 3.4 \mathrm{~cm}$ & * & * & * \\
\hline \multicolumn{7}{|c|}{ Root Width } \\
\hline & \multicolumn{3}{|c|}{ Horizontal to Surface } & \multicolumn{3}{|c|}{ Vertical to Surface } \\
\hline & Small & Medium & Large & Small & Medium & Large \\
\hline & RMSE SD & RMSE SD & RMSE SD & RMSE SD & RMSE SD & RMSE SD \\
\hline Angle & $4.0 \mathrm{~cm} \pm 2.1 \mathrm{~cm}$ & $3.0 \mathrm{~cm} \pm 3.5 \mathrm{~cm}$ & $7.4 \mathrm{~cm} \pm 4.0 \mathrm{~cm}$ & $5.0 \mathrm{~cm} \pm 3.1 \mathrm{~cm}$ & $6.2 \mathrm{~cm} \pm 2.0 \mathrm{~cm}$ & $9.2 \mathrm{~cm} \pm 2.0 \mathrm{~cm}$ \\
\hline Parallel & $16.7 \mathrm{~cm} \pm 7.0 \mathrm{~cm}$ & $20.9 \mathrm{~cm} \pm 3.5 \mathrm{~cm}$ & $11.0 \mathrm{~cm} \pm 4.7 \mathrm{~cm}$ & $*$ & $*$ & $*$ \\
\hline Perpendicular & $7.4 \mathrm{~cm} \pm 1.5 \mathrm{~cm}$ & $8.7 \mathrm{~cm} \pm 0.6 \mathrm{~cm}$ & $7.9 \mathrm{~cm} \pm 2.5 \mathrm{~cm}$ & * & * & * \\
\hline
\end{tabular}

VV: vertical transmit and vertical receive; $\mathrm{HH}$ : horizontal transmit and horizontal receive.

The results in Table 3 would suggest that the VV polarization is the most effective at accurately characterizing root shape across multiple root orientations. Though VV was the most accurate at detecting across root orientations, it did, however, demonstrate inaccuracies and, in some instances, was not capable of discerning root features. This was evident for (1) Root features that are parallel to the scan direction of the antenna, however, as expected, VV clearly outperformed HH and (2) For features that are vertically oriented to the surface and parallel to scan direction.

\subsection{Depth}

In the polarization results, it was evident that the vertical to surface orientation influenced the accuracy of the data. Previous literature [22] has suggested that depth would have a negative effect on root characterization. To validate this, the analysis was done for roots buried vertical to the surface. Each root had three width measures taken. Each width would be positioned at varying depths based on the downward angle of the buried root feature. Based on the findings of cross-line spacing and polarization, the optimal data set was utilized for this analysis. Specifically, data acquired at $2.5 \mathrm{~cm}$ cross-line spacing with the VV polarization antenna was utilized. Also, only data acquired in which the root ran perpendicular to the scan direction was utilized. This was because the overall precision for this data was best. This would allow for the optimal estimation effect by the depth to be achieved. The results for the depth analysis can be found in Table 4 . 
Table 4. Depth analysis in which data was captured at $2.5 \mathrm{~cm}$ cross-line spacing using a VV polarization for roots that were vertical to surface and perpendicular to scan direction. This was done for the three size classes of small, medium, and large.

\begin{tabular}{|c|c|c|c|}
\hline \multicolumn{4}{|c|}{ Depth Analysis } \\
\hline & Small & Medium & Large \\
\hline & RMSE SD & RMSE SD & RMSE SD \\
\hline Width 1 & $3.1 \mathrm{~cm} \pm 1.0 \mathrm{~cm}$ & $5.0 \mathrm{~cm} \pm 3.1 \mathrm{~cm}$ & $5.8 \mathrm{~cm} \pm 1.5 \mathrm{~cm}$ \\
\hline Width 2 & $4.8 \mathrm{~cm} \pm 1.5 \mathrm{~cm}$ & $6.2 \mathrm{~cm} \pm 2.0 \mathrm{~cm}$ & $13.1 \mathrm{~cm} \pm 1.7 \mathrm{~cm}$ \\
\hline Width 3 & $3.7 \mathrm{~cm} \pm 2.6 \mathrm{~cm}$ & $9.1 \mathrm{~cm} \pm 2.0 \mathrm{~cm}$ & $20.8 \mathrm{~cm} \pm 3.1 \mathrm{~cm}$ \\
\hline
\end{tabular}

The results from Table 4 would suggest that depth does have a significant effect on characterizing root shape. However, this was only evident for medium to large roots as small roots did not exhibit the same trend of accuracy degradation with depth.

\section{Discussion}

The objective of this research was to determine the functionality of currently available antenna design for the detection and parameterization of root architecture to be utilized for estimating marketable product, more specifically, root shape. The system was to be tested across multiple root orientations and dimensions to define the limitations and capabilities of the system with modified acquisition methodologies. The long-term objective of the study is the optimization of currently available sensor platforms for the use in root architecture mapping, which can be transferable to breeding programs for developing new cultivars that have more marketable root traits and a decision support platform for large scale farming in delivering marketable goods at an earlier delivery point.

The results demonstrated highly accurate capabilities of the sensor when roots ran at a perpendicular or 45-degree angle to the scan line. This result was also confirmed by Cui et al., [21] in which they found an average $R^{2}$ value of 0.86 with an average root mean squared error (RMSE) of $0.36 \mathrm{~cm}$ for root width at two locations. Though the method utilized in this study was not the same, the results of each method would provide validity to the ability of GPR to detect root architecture. This is only evident for certain root orientations, however. Roots ran parallel to the scan direction created inaccurate results. We attribute this issue to two-dimensional migration. Since the migration functions performed on the data were only performed in two dimensions, the function would not collapse the signal from the opposite direction. This migration is done solely on the radargram in the scan direction. Since the root feature would cover multiple radargrams across scan lines, it is expected that the feature would appear in scan lines beyond the root dimension limits due to the emitted radiation pattern that would capture responses from further than the root feature. This creates a false feature at greater distances perpendicular to the root feature since the signal was not migrated in that cross direction and, therefore, increases the dimension of the root. The expected solution to this issue would be utilizing a more computationally intensive three-dimensional migration function.

When characterizing root features for roots with an inclination of 45-degrees from the surface, there were discrepancies in the accuracy of the measures. Root features were not evident at all when utilizing a root orientation parallel to the scan direction which could be attributed again to the migration issue. However, in this case, the widebody reconstruction of the root features began to merge into one another after interpolation which did not allow for estimation of dimensions. This issue is clearly evident in Figure 7. Though migration is expected to be a factor in this scenario, it can also be inferred that interpolation would have created distortions in the images. When conducting interpolation, an inverse distance weighted function is utilized. Based on spatial autocorrelation, it would be expected that the interpolation would over enhance these larger features in the parallel direction while reducing the overlap when the spacing between features is increased. A factor that can also be contributing to these merged features would be depth. As found in Depth Analysis shown 
in Table 4, the greater the depth, the less accurate the measure becomes. This could suggest that the deeper root features are characterized to a larger dimension creating overlap and, therefore, loss of clarity when creating isosurfaces.

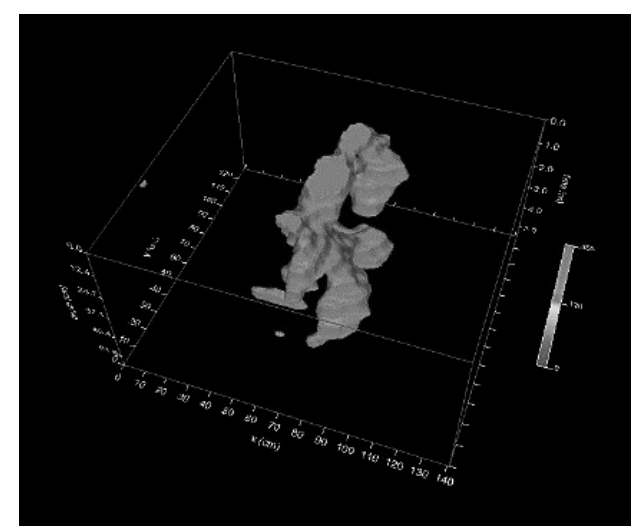

Figure 7. Illustration of isosurfaces in which no root features could be identified.

The VV polarization suggested optimal detection capabilities as found in the polarization results in Table 3. However, theoretical knowledge would suggest that for root detection perpendicular to scan line direction, an $\mathrm{HH}$ antenna would have the best results. This is validated in Simi et al. [37]. In this study, the same sensor concept of dual polarization and frequency was utilized, and results suggested that the $\mathrm{HH}$ polarization had the best detection capabilities of rebar running perpendicular to the scan line direction. However, a dense array of VV sensors have already been proven at producing outstanding results in mapping underground utilities running perpendicular to the scan direction [38,39]. These are particularly encouraging results as VV polarization permits building denser arrays, which may be necessary for characterizing small roots features.

A statistic that was also of interest and would be introduced for discussion in this article is the accuracy of the sensor in relation to root features that run at a 45-degree angle to the scan direction. Theoretically, a cross polarization antenna would be the best approach for acquisition of this type of feature; however, both the VV and $\mathrm{HH}$ polarizations exhibited high accuracy and, in some cases, better accuracy than that of a feature most expected to exhibit greater detection. When collecting data with a horizontal polarization antenna on a feature that is moving perpendicular to the scan direction, the $\mathrm{HH}$ polarization would be expected to perform best on this feature. However, the $\mathrm{HH}$ polarization was found to be more accurate on the feature that runs at an angle to the scan line. This result is of interest and should be studied further to determine if the effect can be attributed to pre-processing methods or interpolation methods.

Though the results suggest positive findings, it should be communicated that limitations still exist for full field deployment. Current antenna designs are not suitable for rugged agricultural conditions creating a constraint in data capture. Field deployed systems must also consider the limitations of the environment. This could include soil variability and moisture [40-43]. The system utilized in this study allows for high spatial resolution; however, in the field conditions expected in true agricultural fields, the chance for increased energy dissipation would be expected.

In conclusion, based on these findings, it can be inferred that the currently available C-Thrue sensor by IDS GeoRadar and GPR-Slice software would allow for accurate estimation of root metrics. The combination of dual polarization is effective for the omnidirectional nature of roots. The findings in this study would provide context for future development and begin a dialogue for the future applications of GPR in agricultural applications in which the opportunity for root characterization is considered a new frontier for development. 
Author Contributions: Conceptualization, A.D., A.N., and D.B.H.; Data curation, A.D.; Formal analysis, A.D. and A.N.; Funding acquisition, D.B.H.; Investigation, A.D. and A.N.; Methodology, A.D., A.N., and D.B.H.; Project administration, A.N. and D.B.H.; Resources, A.N. and D.B.H.; Software, A.D.; Supervision, A.N. and D.B.H.; Validation, A.D. and A.N.; Visualization, A.D.; Writing-original draft, A.D. and A.N.; Writing-review and editing, A.D., A.N., and D.B.H.

Funding: This research was funded by the National Science Foundation, grant number 1543957.

Conflicts of Interest: The authors declare no conflict of interest. The funders had no role in the design of the study; in the collection, analyses, or interpretation of data; in the writing of the manuscript, or in the decision to publish the results.

\section{References}

1. Allem, A.C. The Origins and Taxonomy of Cassava, in Cassava: Biology, Production and Utilization; Hillcocks, R.J., Thresh, J.M., Bellotti, A.C., Eds.; CABI Publishing: New York, NY, USA, 2002; pp. 1-16.

2. Nassar, N.; Ortiz, R. Breeding cassava to feed the poor. Sci. Am. 2010, 302, 78-84. [CrossRef] [PubMed]

3. Ceballos, H.; Ramirez, J.; Bellotti, A.C.; Jarvis, A.; Alvarez, E. Adaptation of cassava to changing climates. In Crop Adaptation to Climate Change; Blackwell Publishing Ltd.: Hoboken, NJ, USA, 2011; pp. 411-425.

4. FAO. FAOSTAT_Country/Territorial Notes: Yield of Commodity. [Database] 2015; Group-Production, Domain-Crops, Area-Africa and South Eastern Asia, Item-Cassava, Element-Yield]. Available online: http://faostat3.fao.org/compare (accessed on 29 March 2016).

5. Ceballos, H.; Hershey, C.; Becerra-López-Lavalle, L.A. New approaches to cassava breeding. Plant Breed. Rev. 2012, 36, 427-504.

6. Okogbenin, E.; Setter, T.L.; Ferguson, M.; Mutegi, R.; Ceballos, H.; Olasanmi, B.; Fregene, M. Phenotypic approaches to drought in cassava: Review. Front. Physiol. 2013, 4, 93. [CrossRef] [PubMed]

7. Ceballos, H.; Hershey, C.H. Cassava (Manihot esculenta Crantz). In Genetic Improvement of Tropical Crops; Springer: Cham, Switzerland, 2017; pp. 129-180.

8. Majdi, H. Root sampling methods-applications and limitations of the minirhizotron technique. Plant Soil 1996, 185, 255-258. [CrossRef]

9. Perret, J.S.; Al-Belushi, M.E.; Deadman, M. Non-destructive visualization and quantification of root using computed tomography. Soil Biol. Biochem. 2007, 39, 391-399. [CrossRef]

10. Metzner, R.; Eggert, A.; van Dusschoten, D.; Pflugfelder, D.; Gerth, S.; Schurr, U.; Uhlmann, N.; Jahnke, S. Direct comparison of MRI and X-ray CT technologies for 3D maging of root systems in soil: Potential and challenges for root trait quantification. Plant Methods 2015, 11, 17. [CrossRef]

11. Al-Nuaimy, W.; Huang, Y.; Nakhkash, M.; Fang, M.T.C.; Nguyen, V.T.; Eriksen, A. Automatic detection of buried utilities and solid objects with GPR using neural networks and pattern recognition. J. Appl. Geophys. 2000, 43, 157-165. [CrossRef]

12. Frigui, H.; Gader, P. Detection and discrimination of land mines in ground-penetrating radar based on edge histogram descriptors and a possibilistic-nearest neighbor classifier. IEEE Trans. Fuzzy Syst. 2009, 17, 185-199. [CrossRef]

13. Ho, K.; Carin, L.; Gader, P.D.; Wilson, J.N. An investigation of using the spectral characteristics from ground penetrating radar for landmine/clutter discrimination. IEEE Trans. Geosci. Remote Sens. 2008, 46, 1177-1191. [CrossRef]

14. Pasolli, E.; Melgani, F.; Donelli, M.; Attoui, R.; De Vos, M. Automatic detection and classification of buried objects in GPR images using genetic algorithms and support vector machines. In Proceedings of the IGARSS 2008-2008 IEEE International Geoscience and Remote Sensing Symposium, Boston, MA, USA, 7-11 July 2008.

15. Andrén, O.; Rajkai, K.; Kätterer, T. A non-destructive technique for studies of root distribution in relation to soil moisture. Agric. Ecosyst. Environ. 1991, 34, 269-278. [CrossRef]

16. Bassuk, N.; Grabosky, J.; Mucciardi, A.; Raffel, G. Ground-penetrating radar accurately locates tree roots in two soil media under pavement. Arboric. Urban For. 2011, 37, 160-166.

17. Butnor, J.; Pruyn, M.L.; Shaw, D.C.; Harmon, M.E.; Mucciardi, A.N.; Ryan, M.G. Detecting defects in conifers with ground penetrating radar: Applications and challenges. For. Pathol. 2009, 39, 309-322. [CrossRef] 
18. Butnor, J.R.; Barton, C.; Day, F.P.; Johnsen, K.H.; Mucciardi, A.N.; Schroeder, R.; Stover, D.B. Using Ground Penetrating to Detect Tree Roots and Estimate Biomass. In Measuring Roots: An Updated Approach; Mancuso, S., Ed.; Springer: New York, NY, USA, 2011; pp. 213-246.

19. Butnor, J.R.; Doolittle, J.A.; Johnsen, K.H.; Samuelson, L.; Stokes, T.; Kress, L. Utility of ground-penetrating radar as a root biomass survey tool in forest systems. Soil Sci. Soc. Am. J. 2003, 67, 1607-1615. [CrossRef]

20. Butnor, J.R.; Doolittle, J.A.; Kress, L.; Cohen, S.; Johnsen, K.H. Use of ground-penetrating radar to study tree roots in the southeastern United States. Tree Physiol. 2001, 21, 1269-1278. [CrossRef]

21. Cui, X.; Chen, J.; Shen, J.; Cao, X.; Chen, X.; Zhu, X. Modeling tree root diameter and biomass by ground-penetrating radar. Sci. China Earth Sci. 2011, 54, 711-719. [CrossRef]

22. Cui, X.; Guo, L.; Chen, J.; Chen, X.; Zhu, X. Estimating tree-root biomass in different depths using ground-penetrating radar: Evidence from a controlled experiment. IEEE Trans. Geosci. Remote Sens. 2013, 51, 3410-3423. [CrossRef]

23. Guo, L.; Lin, H.; Fan, B.; Cui, X.; Chen, J. Forward simulation of root's ground penetrating radar signal: Simulator development and validation. Plant Soil 2013, 372, 487-505. [CrossRef]

24. Guo, L.; Lin, H.; Fan, B.; Cui, X.; Chen, J. Impact of root water content on root biomass estimation using ground penetrating radar: Evidence from forward simulations and field controlled experiments. Plant Soil 2013, 371, 503-520. [CrossRef]

25. Hirano, Y.; Dannoura, M.; Aono, K.; Igarashi, T.; Ishii, M.; Yamase, K.; Makita, N.; Kanazawa, Y. Limiting factors in the detection of tree roots using ground-penetrating radar. Plant Soil 2009, 319, 15-24. [CrossRef]

26. Molon, M.M. Quantitative, Non-Destructive Estimates of Forest Coarse Root Biomass Using 3-D Ground-Penetrating Radar (GPR). Ph.D. Thesis, McMaster University, Hamilton, ON, Canada, 2012.

27. Raz-Yaseef, N.; Koteen, L.; Baldocchi, D.D. Coarse root distribution of a semi-arid oak savanna estimated with ground penetrating radar. J. Geophys. Res. Biogeosci. 2013, 118, 135-147. [CrossRef]

28. Zhu, S.; Huang, C.; Su, Y.; Sato, M. 3D Ground Penetrating Radar to Detect Tree Roots and Estimate Root Biomass in the Field. Remote Sens. 2014, 6, 5754-5773. [CrossRef]

29. Delgado, A.; Hays, D.B.; Bruton, R.K.; Ceballos, H.; Novo, A.; Boi, E.; Selvaraj, M.G. Ground penetrating radar: A case study for estimating root bulking rate in cassava (Manihot esculenta Crantz). Plant Methods 2017, 13, 65. [CrossRef]

30. Daniels, D.J. Ground penetrating radar. In Encyclopedia of RF and Microwave Engineering; IET: Hoboken, NJ, USA, 2004; Volume 1.

31. Jol, H.M. Ground Penetrating Radar Theory and Applications; Elsevier: Amsterdam, The Netherlands, 2008.

32. Pasculli, D.; Natali, A.; Salvatore, W.; Morelli, F.; Morandi, D. Investigation of reinforced concrete bridges by using a dual-polarized high-frequency GPR. In Proceedings of the 2018 17th International Conference on Ground Penetrating Radar (GPR), Rapperswil, Switzerland, 18-21 June 2018.

33. Grasmueck, M.; Novo, A. 3D GPR imaging of shallow plastic pipes, tree roots, and small objects. In Proceedings of the 2016 16th International Conference on Ground Penetrating Radar (GPR), Hong Kong, China, 13-16 June 2016.

34. Grasmueck, M.; Weger, R.; Horstmeyer, H.J.G. Full-resolution 3D GPR imaging. Geophysics 2005, 70, K12-K19. [CrossRef]

35. Goodman, D. GPR-SLICE Software; Geophysical Archaeometry Laboratory Inc.: Woodland Hills, CA, USA, 2016.

36. Autodesk, Inc. AutoCAD 2012; Autodesk, Inc.: San Rafael, CA, USA, 2011.

37. Simi, A.; Manacorda, A. Benedetto. Bridge deck survey with high resolution ground penetrating radar. In Proceedings of the 2012 14th International Conference on Ground Penetrating Radar (GPR), Shanghai, China, 4-8 June 2012.

38. Pasculli, D.; Giannino, F.; Simi, A.; Manacorda, G. High-density dual polarized GPR array for underground asset mapping. In Proceedings of the 2017 9th International Workshop on Advanced Ground Penetrating Radar (IWAGPR), Edinburgh, UK, 28-30 June 2017.

39. Simi, A.; Manacorda, G.; Miniati, M.; Bracciali, S.; Buonaccorsi, A. Underground asset mapping with dual-frequency dual-polarized GPR massive array. In Proceedings of the XIII International Conference on Ground Penetrating Radar (GPR), Lecce, Italy, 21-25 June 2012. 
40. Detsch, R.M.; Jenkins, T.F.; Arcone, S.A.; Koh, G.; O’Neill, K. Environmental effects on detection of buried mines and UXO. In Detection and Remediation Technologies for Mines and Minelike Targets III; Aerospace/Defense Sensing and Controls; Intermational Society for Optics and Photonics: Bellingham, WA, USA, 1998.

41. Doolittle, J.A.; Collins, M.E. Use of soil information to determine application of ground penetrating radar. J. Appl. Geophys. 1995, 33, 101-108. [CrossRef]

42. Igel, J. The small-scale variability of electrical soil properties-influence on GPR measurements. In Proceedings of the 12th International Conference on Ground Penetrating Radar, Birmingham, UK, 16-19 June 2008.

43. Miller, T.W.; Borchers, B.; Hendrickx, J.M.; Hong, S.; Dekker, L.W.; Ritsema, C.J. Effects of soil physical properties on GPR for landmine detection. In Proceedings of the Fifth International Symposium on Thecnology and the Mine Problem, Monterey, CA, USA, 22-25 April 2002.

(C) 2019 by the authors. Licensee MDPI, Basel, Switzerland. This article is an open access article distributed under the terms and conditions of the Creative Commons Attribution (CC BY) license (http://creativecommons.org/licenses/by/4.0/). 